\title{
A classical long-time tail in a driven granular fluid
}

\author{
W T Kranz \\ Georg-August-Universität Göttingen, Institut für Theoretische Physik, \\ Friedrich-Hund-Platz 1, 37077 Göttingen, Germany \\ Max-Planck-Institut für Dynamik und Selbstorganisation, Am Faßberg 17, 37077 \\ Göttingen, Germany \\ E-mail: kranz@theorie.physik.uni-goettingen.de
}

\begin{abstract}
I derive a mode-coupling theory for the velocity autocorrelation function, $\psi(t)$, in a fluid of randomly driven inelastic hard spheres far from equilibrium. With this, I confirm a conjecture from simulations that the velocity autocorrelation function decays algebraically, $\psi(t) \propto t^{-3 / 2}$, if momentum is conserved. I show that the slow decay is due to the coupling to transverse currents.

PACS numbers: 47.57.Gc, 51.10.+y
\end{abstract}

Submitted to: J. Phys.: Condens. Matter

\section{Introduction}

The algebraic, rather than exponential in time decay of the velocity autocorrelation function (VACF), $\psi(t) \propto t^{-\alpha}$, in simple fluids had been quite a surprise when it was first discovered [1, 2]. It was finally explained by mode coupling theories and attributed to vortex flows [3]. Long-time tails are expected even in high energy physics [4] now and have been reported recently also for fluids far from equilibrium [5]. In particular they are discussed for granular fluids [6, 7, 8, 9, 10, 11].

Here, I show that the original mode coupling argument [12, 13, 14] can be adapted to the stationary state of a randomly driven granular fluid. In particular, I explain the observation that $\alpha \approx 1.5$ in three space dimensions [11]. This is exactly the same exponent as for equilibrium fluids and stands in contrast to a number of unconventional exponents reported in the literature.

In a fluid in thermal equilibrium, long-time tails are a result of the coupling to the transverse current modes, $\boldsymbol{j}_{\boldsymbol{k}}^{\mathrm{T}}$, labelled by the wave vector $\boldsymbol{k}$. A number of approaches (see [3] and references therein, and [13, 14, 15, 16, 17]) confirmed the result $\psi(t \rightarrow \infty) \propto t^{-3 / 2}$. In a Lorentz gas, momentum is not conserved and it was argued [18] that this leads to a faster decay, $\psi(t \rightarrow \infty) \propto t^{-5 / 2}$. See [19] for why this behaviour may be hard to observe. 
For a freely cooling granular gas, a long-time tail in the number of collisions, $\tau$, is predicted of the form $\psi(\tau \rightarrow \infty) \propto \tau^{-3 / 2}$ [7]. Here, the coupling to the longitudinal and transverse current are both relevant. For shear-driven granular fluids, there are two competing proposals. Hayakawa and Otsuki [8] predict $\psi(t \rightarrow \infty) \propto(\dot{\gamma} t)^{-5 / 2}$, where $\dot{\gamma}$ is the shear rate and Kumaran [10] predicts $\psi(t \rightarrow \infty) \propto(\dot{\gamma} t)^{-7 / 2}$ in the vorticity direction and a slightly faster decay, $\psi(t \rightarrow \infty) \propto(\dot{\gamma} t)^{-15 / 4}$, in the gradient and flow directions. The difference remains unresolved [20]. In both theories, the physical interpretation of the relevant collective modes is not obvious.

From the above discussion one can conclude that the existence of long-time tails seems to be rather universal even in fluids far from equilibrium. Two questions, however, have to be answered for every specific system: What is the mechanism that induces the slow decay and what is the value of the exponent $\alpha$ ? In the following, I will address these two questions for the randomly driven granular fluid.

The paper is organised as follows. We start in section 2 by defining our model system. In section $3 \mathrm{I}$ give the formally exact equation of motion for the VACF. This will be closed in section 4 by a mode-coupling approximation. In section 5 I discuss the results of the approximation. In particular, the long-time tails. In the final section 6 I summarise my results and give some perspectives for future work.

\section{Model}

\subsection{Inelastic Hard Spheres}

The granular fluid is modelled as a monodisperse system of $N$ smooth inelastic hard spheres of diameter $d$ and mass $m=1$ in a volume $V=L^{3}$. I consider the thermodynamic limit $N, V \rightarrow \infty$ such that the density $n=N / V$ remains finite. Dissipation is introduced through a constant coefficient of normal restitution $\varepsilon \in[0,1]$ that augments the law of reflection [21],

$$
\hat{\boldsymbol{r}}_{12} \cdot \boldsymbol{v}_{12}^{\prime}=-\varepsilon \hat{\boldsymbol{r}}_{12} \cdot \boldsymbol{v}_{12}
$$

where $\boldsymbol{v}_{12}=\boldsymbol{v}_{1}-\boldsymbol{v}_{2}$ is the relative velocity and $\hat{\boldsymbol{r}}_{12}$ is the unit vector pointing from the centre of particle 2 to particle 1 . The prime indicates post-collisional quantities.

\subsection{Stochastic Driving Force}

The driving force is implemented as an external random force,

$$
\boldsymbol{v}_{i}^{\prime}(t)=\boldsymbol{v}_{i}(t)+\sqrt{P_{\mathrm{D}}} \boldsymbol{\xi}_{i}(t),
$$

where $P_{\mathrm{D}}$ is the driving power. The $\xi_{i}^{\alpha}, \alpha=x, y, z$ are Gaussian random variables with zero mean and variance,

$$
\left\langle\xi_{i}^{\alpha}(t) \xi_{j}^{\beta}\left(t^{\prime}\right)\right\rangle_{\xi}=\left[\delta_{i j}-\delta_{\pi(i), j}\right] \delta^{\alpha \beta} \delta\left(t-t^{\prime}\right)
$$

where $\pi(i)$ denotes the nearest neighbour of particle $i$. In effect, the two particles $i$ and $\pi(i)$ are driven by forces of equal strength but opposite direction. Thereby, the external force does not destroy momentum conservation on macroscopic length scales [11, 22]. 


\subsection{The Granular Fluid}

Macroscopically, the fluid is fully characterised by the packing fraction, $\varphi=\pi n d^{3} / 6$, the coefficient of restitution, $\varepsilon$, and the driving power, $P_{\mathrm{D}}$. In the stationary state, the granular temperature $T=T\left(\varphi, \varepsilon, P_{\mathrm{D}}\right)=\frac{1}{3 N} \sum_{i} \boldsymbol{v}_{i}^{2}$ is given by the balance between the driving power, $P_{\mathrm{D}}$, and the energy loss through the inelastic collisions.

The collision frequency $\omega_{c} \propto \sqrt{T}$ is the only time scale of the system. Thus, changing the granular temperature only changes the time scale of the system. I use this freedom and set $T \equiv 1$ in the following.

\section{Microscopic Description}

\subsection{Phase Space Distribution}

In contrast to fluids in thermal equilibrium, no analytical expression for the stationary phase space distribution of driven granular fluids is known so far. Therefore, I have to make a few assumptions to evaluate the expectation values. First of all I assume that positions and velocities are uncorrelated, $\varrho(\Gamma)=\varrho_{\mathrm{r}}\left(\left\{\boldsymbol{r}_{i}\right\}\right) \varrho_{\mathrm{v}}\left(\left\{\boldsymbol{v}_{i}\right\}\right)$. Moreover, I assume that the velocity distribution factorises into a product of one particle distribution functions, $\varrho_{\mathrm{v}}\left(\left\{\boldsymbol{v}_{i}\right\}\right)=\prod_{i} \varrho_{1}\left(\boldsymbol{v}_{i}\right)$. All we need to know about $\varrho_{1}(\boldsymbol{v})$ are a few moments. Namely, that it has a vanishing first moment, $\int \mathrm{d}^{3} v \boldsymbol{v} \varrho_{1}(\boldsymbol{v})=0$, a finite second moment, $\int \mathrm{d}^{3} v v^{2} \varrho_{1}(\boldsymbol{v})=3 T<\infty$ and a finite third collisional moment, $\int \mathrm{d}^{3} v(\hat{\boldsymbol{r}} \cdot \boldsymbol{v})^{3} \Theta(-\hat{\boldsymbol{r}} \cdot \boldsymbol{v}) \varrho_{1}(\boldsymbol{v})<\infty$. The spatial distribution function, $\varrho_{\mathrm{r}}\left(\left\{\boldsymbol{r}_{i}\right\}\right)$, enters the theory via static correlation function, as will be discussed below.

Averages over pairs of observables define a scalar product, $\langle A \mid B\rangle:=\left\langle A^{*} B\right\rangle:=$ $\int \mathrm{d} \Gamma \varrho(\Gamma) A^{*}(\Gamma) B(\Gamma)$, where $A^{*}$ denotes the complex conjugate of $A$.

\subsection{Observables}

The VACF, $\psi(t)=\left\langle\boldsymbol{v}_{s} \mid \boldsymbol{v}_{s}(t)\right\rangle / 3$, is defined in terms of the tagged particle velocity $\boldsymbol{v}_{s}$. The tagged particle position will be described by the density $\rho^{s}(\boldsymbol{r}, t)=\delta\left(\boldsymbol{r}_{s}-\boldsymbol{r}(t)\right)$. The host fluid is characterised by the density and current fields

$$
\begin{aligned}
& \rho(\boldsymbol{r}, t)=\frac{1}{N} \sum_{i} \delta\left(\boldsymbol{r}-\boldsymbol{r}_{i}(t)\right), \\
& \boldsymbol{j}(\boldsymbol{r}, t)=\frac{1}{N} \sum_{i} \boldsymbol{v}_{i}(t) \delta\left(\boldsymbol{r}-\boldsymbol{r}_{i}(t)\right) .
\end{aligned}
$$

In particular, I will use the spatial Fourier transform of those fields, $\rho_{\boldsymbol{k}}^{s}(t), \rho_{\boldsymbol{k}}(t)$, and the longitudinal and transverse current fields $j_{\boldsymbol{k}}^{L}(t)=\hat{\boldsymbol{k}} \cdot \boldsymbol{j}_{\boldsymbol{k}}(t)$, and $\boldsymbol{j}_{\boldsymbol{k}}^{T}(t)=\boldsymbol{j}_{\boldsymbol{k}}(t)-\hat{\boldsymbol{k}} j_{\boldsymbol{k}}^{L}(t)$, respectively.

\subsection{Dynamics}

We have shown in [23] that the time evolution operator $\mathrm{U}(t)=\exp \left(\mathrm{i} t \mathcal{L}_{+}\right)$can be written in terms of an effective pseudo Liouville operator $\mathcal{L}_{+}[24]$. It is given as a sum of three 
parts, $\mathcal{L}_{+}=\mathcal{L}_{0}+\mathcal{T}_{+}+\mathcal{L}_{\mathrm{D}}^{+}$, which are in turn: The free streaming operator $\mathcal{L}_{0}$, the collision operator $\mathcal{T}_{+}$, and the driving operator $\mathcal{L}_{\mathrm{D}}^{+}$.

With the Mori projectors $\mathcal{P}=\left|\boldsymbol{v}_{s}\right\rangle\left\langle\boldsymbol{v}_{s}\right| / 3, \mathcal{Q}=1-\mathcal{P}$, one can derive a formally exact equation of motion for the $\mathrm{VACF}$

$$
\dot{\psi}(t)+\frac{1+\varepsilon}{3} \omega_{\mathrm{E}} \psi(t)+\omega_{\mathrm{E}}^{2} \int_{0}^{t} \mathrm{~d} \tau m(t-\tau) \psi(\tau)=0,
$$

where the local term $\left\langle\boldsymbol{v}_{s} \mid i \mathcal{L}_{+} \boldsymbol{v}_{s}\right\rangle / 3=-(1+\varepsilon) \omega_{\mathrm{E}} / 3$ was determined in [1]. The memory kernel is formally given as

$$
m(t)=\left\langle\boldsymbol{v}_{s} \mid \mathcal{L}_{+} \mathcal{Q} \tilde{\mathrm{U}}(t) \mathcal{Q} \mathcal{L}_{+} \boldsymbol{v}_{s}\right\rangle / 3 \omega_{\mathrm{E}}^{2}
$$

and $\tilde{U}(t)=\exp \left(\mathrm{it} \mathcal{Q} \mathcal{L}_{+} \mathcal{Q}\right)$ is a modified propagator [25, 26, 23]. The Enskog collision frequency $\omega_{\mathrm{E}}=24 \varphi \chi / \sqrt{\pi} d$ is given in terms of the contact value of the pair correlation function at contact, $\chi[27$.

\section{Mode-Coupling Approximations}

I consider three contributions to the memory kernel $m(t) \approx m_{\rho}(t)+m_{\mathrm{L}}(t)+m_{\mathrm{T}}(t)$ that are induced by the coupling of the tagged particle to the host fluid. Namely, to the collective density field $\left[m_{\rho}(t)\right]$, and to the longitudinal and transverse current field $\left[m_{\mathrm{L}}(t)\right.$ and $m_{\mathrm{T}}(t)$, respectively].

The behaviour of the collective modes is characterised by their two-point correlation functions,

$$
\begin{aligned}
& \phi(k, t)=N\left\langle\rho_{\boldsymbol{k}} \mid \rho_{\boldsymbol{k}}(t)\right\rangle / S_{k}, \\
& \phi_{\mathrm{L}}(k, t)=N\left\langle j_{\boldsymbol{k}}^{\mathrm{L}} \mid j_{\boldsymbol{k}}^{\mathrm{L}}(t)\right\rangle, \\
& \phi_{\mathrm{T}}^{\alpha \beta}(k, t)=N\left\langle j_{\boldsymbol{k}}^{\mathrm{T} \alpha} \mid j_{\boldsymbol{k}}^{\mathrm{T} \beta}(t)\right\rangle=\phi_{\mathrm{T}}(k, t) \delta^{\alpha \beta},
\end{aligned}
$$

where $S_{k}=N\left\langle\rho_{\boldsymbol{k}} \mid \rho_{\boldsymbol{k}}\right\rangle$ is the static structure factor, and

$$
\phi_{\mathrm{s}}(k, t)=\left\langle\rho_{\boldsymbol{k}}^{\mathrm{s}} \mid \rho_{\boldsymbol{k}}^{\mathrm{s}}(t)\right\rangle
$$

is the incoherent scattering function.

In terms of these correlation functions, I replace the modified propagator

$$
\begin{aligned}
\tilde{\mathrm{U}}(t) & \approx N \sum_{\boldsymbol{k}}\left|\rho_{\boldsymbol{k}} \rho_{-\boldsymbol{k}}^{\mathrm{s}}\right\rangle \phi(k, t) \phi_{\mathrm{s}}(k, t)\left\langle\rho_{\boldsymbol{k}} \rho_{-\boldsymbol{k}}^{\mathrm{s}}\right| / S_{k} \\
& +N \sum_{\boldsymbol{k}}\left|j_{\boldsymbol{k}}^{L} \rho_{-\boldsymbol{k}}^{\mathrm{s}}\right\rangle \phi_{\mathrm{L}}(k, t) \phi_{\mathrm{s}}(k, t)\left\langle j_{\boldsymbol{k}}^{L} \rho_{-\boldsymbol{k}}^{\mathrm{s}}\right| \\
& +\frac{N}{2} \sum_{\boldsymbol{k}}\left|j_{\boldsymbol{k}}^{\mathrm{T}} \rho_{-\boldsymbol{k}}^{\mathrm{s}}\right\rangle \phi_{\mathrm{T}}(k, t) \phi_{\mathrm{s}}(k, t)\left\langle j_{\boldsymbol{k}}^{\mathrm{T}} \rho_{-\boldsymbol{k}}^{\mathrm{s}}\right|
\end{aligned}
$$

by a mode-coupling approximation. Similar approximations have been made in, e.g., [12, 13].

The coupling to the collective density field then reads

$$
3 \omega_{\mathrm{E}}^{2} m_{\rho}(t)=\sum_{\boldsymbol{k}} \mathcal{V}_{\boldsymbol{k}}^{\rho} \mathcal{W}_{\boldsymbol{k}}^{\rho} \phi(k, t) \phi_{\mathrm{s}}(k, t)
$$


where the vertices

$$
\begin{aligned}
& \mathcal{V}_{\boldsymbol{k}}^{\rho}=\sqrt{N / S_{k}}\left\langle\boldsymbol{v}_{s} \mid \mathcal{L}_{+} \mathcal{Q} \rho_{\boldsymbol{k}} \rho_{-\boldsymbol{k}}^{\mathrm{s}}\right\rangle=\boldsymbol{k}\left(S_{k}-1\right) / \sqrt{N S_{k}} \\
& \mathcal{W}_{\boldsymbol{k}}^{\rho}=\sqrt{N / S_{k}}\left\langle\rho_{\boldsymbol{k}} \rho_{-\boldsymbol{k}}^{\mathrm{s}} \mid \mathcal{Q} \mathcal{L}_{+} \boldsymbol{v}_{s}\right\rangle=\frac{1+\varepsilon}{2} \boldsymbol{k}\left(S_{k}-1\right) / \sqrt{N S_{k}}
\end{aligned}
$$

can be deduced from equations (46) and (47) in [23]. Explicitly, we find

$$
\omega_{\mathrm{E}}^{2} m_{\rho}(t)=\frac{2 \pi^{2}}{9} \frac{1+\varepsilon}{2} \frac{d^{3}}{\varphi} \int_{0}^{\infty} \frac{\mathrm{d} k k^{4}}{(2 \pi)^{3}} S_{k}\left(n c_{k}\right)^{2} \phi(k, t) \phi_{\mathrm{s}}(k, t),
$$

where $n c_{k}=1-1 / S_{k}$ is the direct correlation function [27]. This implies that $\omega_{\mathrm{E}}^{2} m_{\rho}(t) \equiv m_{0}(t)$, where $m_{0}(t)$ is given in [28] as the memory kernel for the mean square displacement. In [28] we were concerned with the behaviour at high densities close to the glass transition and we used a mode-coupling approximation for the coherent scattering function, $\phi(k, t)$, itself. Here, I am interested in the regime of moderate densities, instead. Consequently, below I will use a hydrodynamic expression for the coherent scattering function [equation $(\underline{20 b})$ ].

The coupling to the currents reads

$$
\begin{aligned}
& 3 \omega_{\mathrm{E}}^{2} m_{\mathrm{L}}(t)=\sum_{\boldsymbol{k}} \mathcal{V}_{\boldsymbol{k}}^{L} \mathcal{W}_{\boldsymbol{k}}^{L} \phi_{\mathrm{L}}(k, t) \phi_{\mathrm{s}}(k, t), \\
& 3 \omega_{\mathrm{E}}^{2} m_{\mathrm{T}}(t)=\frac{1}{2} \sum_{\alpha, \beta} \sum_{\boldsymbol{k}} \mathcal{V}_{\boldsymbol{k}}^{\alpha \beta} \mathcal{W}_{\boldsymbol{k}}^{\alpha \beta} \phi_{\mathrm{T}}(k, t) \phi_{\mathrm{s}}(k, t),
\end{aligned}
$$

where the vertices

$$
\begin{aligned}
& \mathcal{V}_{\boldsymbol{k}}^{\mathrm{L}}=\sqrt{N}\left\langle\boldsymbol{v}_{s} \mid \mathcal{L}_{+} \mathcal{Q} j_{\boldsymbol{k}}^{L} \rho_{-\boldsymbol{k}}^{\mathrm{s}}\right\rangle, \\
& \mathcal{W}_{\boldsymbol{k}}^{\mathrm{L}}=\sqrt{N}\left\langle j_{\boldsymbol{k}}^{L} \rho_{-\boldsymbol{k}}^{\mathrm{s}} \mid \mathcal{Q} \mathcal{L}_{+} \boldsymbol{v}_{s}\right\rangle,
\end{aligned}
$$

and

$$
\begin{aligned}
& \mathcal{V}_{\boldsymbol{k}}^{\alpha \beta}=\sqrt{N}\left\langle v_{s}^{\beta} \mid \mathcal{L}_{+} \mathcal{Q} j_{-\boldsymbol{k}}^{T \alpha} \rho_{\boldsymbol{k}}^{\mathrm{s}}\right\rangle, \\
& \mathcal{W}_{\boldsymbol{k}}^{\alpha \beta}=\sqrt{N}\left\langle j_{-\boldsymbol{k}}^{T \alpha} \rho_{\boldsymbol{k}}^{\mathrm{s}} \mid \mathcal{Q} \mathcal{L}_{+} v_{s}^{\beta}\right\rangle
\end{aligned}
$$

are calculated in Appendix A. While $\mathcal{V}_{\boldsymbol{k}}^{\rho} \neq \mathcal{W}_{\boldsymbol{k}}^{\rho}$ indicates the violation of time reversal invariance in the dissipative fluid, one finds

$$
\begin{aligned}
& \mathcal{V}_{\boldsymbol{k}}^{\mathrm{L}}=\mathcal{W}_{\boldsymbol{k}}^{\mathrm{L}}=\mathrm{i} \frac{1+\varepsilon}{3} \hat{\boldsymbol{k}} \omega_{\mathrm{E}} U_{\mathrm{L}}(k d) / \sqrt{N}, \\
& \mathcal{V}_{\boldsymbol{k}}^{\alpha \beta}=\mathcal{W}_{\boldsymbol{k}}^{\alpha \beta}=\mathrm{i} \sqrt{2 / 3} \delta^{\alpha \beta} \frac{1+\varepsilon}{3} \omega_{\mathrm{E}} U_{\mathrm{T}}(k d) / \sqrt{N},
\end{aligned}
$$

where $U_{\mathrm{L}}(x)=3 j_{0}^{\prime \prime}(x)$ and $U_{\mathrm{T}}(x)=\sqrt{6} j_{0}^{\prime}(x) / x$ are effective potentials. Here, $j_{0}(x)$ is the zeroth order spherical Bessel function [29] and the prime denotes the derivative with respect to the argument. Notably, the effective potentials are independent of density. The vertices are similar in form to those found in [12, 13].

For the memory kernels, we find

$$
m_{\mathrm{L}, \mathrm{T}}(t)=-\frac{8 \pi^{2}}{81} \frac{(1+\varepsilon)^{2}}{4} \frac{d^{3}}{\varphi} \int_{0}^{\infty} \frac{\mathrm{d} k k^{2}}{(2 \pi)^{3}} U_{\mathrm{L}, \mathrm{T}}^{2}(k d) \phi_{\mathrm{L}, \mathrm{T}}(k, t) \phi_{\mathrm{s}}(k, t) .
$$

$\ddagger$ The apparent divergence for $\varphi \rightarrow 0$ is spurious as $\omega_{\mathrm{E}} \sim \mathrm{O}(\varphi)$. 
Given a static structure factor, $S_{k}$, and the dynamic correlator (7a, b,c) and (8) the approximate memory kernel is fully determined by equations (12) and (17). All three contributions to the approximate memory kernel diverge in the short time limit. Actually, the memory kernel should vanish for $t \rightarrow 0$. For elastic hard spheres, a number of proposals to that end have been made [12, 13, 15]. As I am only interested in the asymptotic behaviour, I will not further discuss this divergence.

\section{Discussion}

The long-time asymptotics, $\psi(t \rightarrow \infty)$, are related to the $\operatorname{limit}_{\lim _{s \rightarrow 0}} s \hat{\psi}(s)$ in the Laplace domain \&. For small $s$ we have

$$
\begin{aligned}
s \hat{\psi}(s) & =s\left[-\mathrm{i} \omega_{\mathrm{E}}+s-\omega_{\mathrm{E}}^{2} \hat{m}(s)\right]^{-1} \\
& \simeq \mathrm{i} \frac{s}{\omega_{\mathrm{E}}}+\frac{s^{2}}{\omega_{E}^{2}}-s \hat{m}(s),
\end{aligned}
$$

i.e., $\lim _{s \rightarrow 0} s \hat{\psi}(s)=-\lim _{s \rightarrow 0} s \hat{m}(s)$ or

$$
\psi(t \rightarrow \infty)=-m(t \rightarrow \infty) \text {. }
$$

The long-time tails of the VACF are identical (up to the sign) to those of the associated memory kernel.

At moderate densities, a driven granular fluid is well described by Navier-Stokes order hydrodynamic equations [30, 31]. Consequently, I assume that the dynamic correlation functions take the following form:

$$
\phi_{\mathrm{s}}(k, t)=\mathrm{e}^{-D k^{2} t},
$$

where $D$ is the diffusion coefficient,

$$
\phi(k, t)=\cos (c k t) \mathrm{e}^{-\Gamma k^{2} t}, \quad \phi_{\mathrm{L}}(k, t)=\ddot{\phi}(k, t) / k^{2},
$$

where $c$ is the speed of sound and $\Gamma$ the sound damping constant, and,

$$
\phi_{\mathrm{T}}(k, t)=\mathrm{e}^{-\eta k^{2} t}
$$

with the shear viscosity $\eta$.

All the transport coefficients and the speed of sound are functions of the coefficient of restitution $\varepsilon$. For the diffusion coefficient, Fiege et al. [11] found $D(\varepsilon) \propto 2 /(1+\varepsilon)$. According to van Noije et al. [32] the sound damping constant is given as $\Gamma=\nu+D_{\Gamma}$ where $\nu$ is the kinematic viscosity and $D_{\Gamma}(\varepsilon) \propto 1 /\left(1-\varepsilon^{2}\right)$ is a term peculiar to inelastic fluids. The viscosities $\eta$ and $\nu$ have a more complicated dependence on the degree of dissipation [33]. The speed of sound, $c$, is smaller in a fluid of inelastic compared to elastic hard spheres but only weakly depends on the value of the coefficient of restitution, $\varepsilon[31,32$.

$\S$ I use the convention $\hat{f}(s)=\mathrm{LT}[f](s)=\mathrm{i} \int_{0}^{\infty} f(t) \mathrm{e}^{-\mathrm{i} s t} \mathrm{~d} t$. 
In the long wave length limit $k \rightarrow 0$ it holds that $S_{k}, c_{k} \rightarrow$ const. and $U_{\mathrm{L}}^{2}(k d) \rightarrow 1$, $U_{\mathrm{T}}^{2}(k d) \rightarrow 2 / 3$. In the long-time limit $t \rightarrow \infty$, we thus find

$$
\begin{aligned}
& m_{\mathrm{T}}(t \rightarrow \infty) \simeq-M_{\mathrm{T}}\left[(D+\eta) t / d^{2}\right]^{-3 / 2} \\
& m_{\mathrm{L}}(t \rightarrow \infty) \simeq-M_{\mathrm{L}}\left[(D+\Gamma) t / d^{2}\right]^{-3 / 2} e^{-c^{2} t / 4(D+\Gamma)} \\
& m_{\rho}(t \rightarrow \infty) \simeq M_{\rho}\left[(D+\Gamma) t / d^{2}\right]^{-1 / 2} e^{-c^{2} t / 4(D+\Gamma)}
\end{aligned}
$$

This is the central result of this contribution. The evaluation of $m_{\mathrm{T}}(t \rightarrow \infty)$ is simply a moment of a gaussian integral. The types of integrals that are necessary for the evaluation of $m_{\rho, \mathrm{L}}(t \rightarrow \infty)$ are discussed in Appendix B. Away from the glass transition, $c^{2} / 4(D+\Gamma) \sim \mathrm{O}\left(\omega_{\mathrm{E}}\right)$, i.e., the contributions $m_{\rho, \mathrm{L}}(t)$ decay on a short time scale $\propto \omega_{\mathrm{E}}^{-1}$. The dominant asymptotic contribution is thus $m(t \rightarrow \infty)=m_{\mathrm{T}}(t \rightarrow \infty)$.

The prefactors read explicitly

$$
\begin{aligned}
& M_{\mathrm{T}}=\frac{1}{486 \sqrt{\pi}} \frac{(1+\varepsilon)^{2}}{4 \varphi}, \\
& M_{\mathrm{L}}=\frac{1}{162 \sqrt{\pi}} \frac{(1+\varepsilon)^{2}}{4 \varphi} \frac{c^{2}}{D+\Gamma}, \\
& M_{\rho}=\frac{1}{1152 \sqrt{\pi}} \frac{1+\varepsilon}{2 \varphi} \frac{S_{0}\left(n c_{0}\right)^{2}}{\omega_{\mathrm{E}}^{2} d^{2}} \frac{c^{4}}{(D+\Gamma)^{4}} .
\end{aligned}
$$

Due to the nontrivial dependence of the viscosity, $\eta(\varphi, \varepsilon)$, and the sound damping $\Gamma(\varphi, \varepsilon)$ on the coefficient of restitution, $\varepsilon$, and on the density, $\varphi$, there is no simple trend of $m_{\mathrm{T}, \mathrm{L}, \rho}$ with $\varepsilon$. A reduction of the memory effects compared to fluid of elastic hard spheres, however, can be expected.

From equation (19), it follows that

$$
\psi(t \rightarrow \infty) \simeq M_{\mathrm{T}}\left[(D+\eta) t / d^{2}\right]^{-3 / 2} \propto t^{-3 / 2} .
$$

With this result I have answered both questions from the introduction. We now know the value of the exponent $\alpha$ and which of the possible couplings is relevant.

At high densities, close to the granular glass transition [23], the viscosity, $\eta$, is expected to be large and the long-time tail will be strongly suppressed [34].

\section{Summary \& Perspectives}

I discussed the coupling of the tagged particle velocity to the hydrodynamic modes of the host fluid in the frame work of mode-coupling theory. Considering a randomly driven inelastic hard sphere fluid with local momentum conservation, I found that the VACF decays algebraically, $\psi(t \rightarrow \infty) \propto t^{-\alpha}$, with an exponent $\alpha=3 / 2$. This supports observations from simulations [11]. The relevant process for the algebraic decay is,

both for elastic and inelastic hard spheres, the coupling to the transverse currents. The coupling to the density and longitudinal currents have a finite life time.

The discussion of the VACF in a randomly driven granular fluid without momentum conservation will be left to future work. This could possibly also help to settle the question about the nature of the long-time tails in the sheared granular fluid. 


\section{Acknowledgments}

I would like to express my gratitude to Annette Zippelius for initiating this study and for long time support. I thank Andrea Fiege and Matthias Sperl for many illuminating discussions and Matthias for critically reading the manuscript.

\section{Appendix A. Vertices}

Here, I will detail the calculation of the vertices.

\section{Appendix A.1. Longitudinal}

Due to the symmetry of the velocity distribution function, we have

$$
\begin{aligned}
\left\langle\boldsymbol{v}_{s} \mid \mathcal{L}_{+} \mathcal{Q} j_{-\boldsymbol{k}}^{\mathrm{L}} \rho_{\boldsymbol{k}}^{\mathrm{s}}\right\rangle & =\left\langle\boldsymbol{v}_{s} \mid \rho_{\boldsymbol{k}}^{\mathrm{s}} \mathcal{T}_{+} j_{-\boldsymbol{k}}^{\mathrm{L}}\right\rangle-\frac{1}{3}\left\langle\boldsymbol{v}_{s} \mid \mathcal{T}_{+} \boldsymbol{v}_{s}\right\rangle\left\langle\boldsymbol{v}_{s} \mid j_{-\boldsymbol{k}}^{\mathrm{L}} \rho_{\boldsymbol{k}}^{\mathrm{s}}\right\rangle \\
& =\hat{\boldsymbol{k}}\left\langle j_{\boldsymbol{k}}^{\mathrm{sL}} \mid \mathcal{T}_{+} j_{\boldsymbol{k}}^{\mathrm{L}}\right\rangle-\mathrm{i} \frac{1+\varepsilon}{3} \omega_{\mathrm{E}} \hat{\boldsymbol{k}}\left\langle j_{\boldsymbol{k}}^{\mathrm{sL}} \mid j_{\boldsymbol{k}}^{\mathrm{L}}\right\rangle
\end{aligned}
$$

and

$$
\begin{aligned}
\left\langle j_{-\boldsymbol{k}}^{\mathrm{L}} \rho_{\boldsymbol{k}}^{\mathrm{s}} \mathcal{Q} \mid \mathcal{L}_{+} \boldsymbol{v}_{s}\right\rangle & =\left\langle j_{-\boldsymbol{k}}^{\mathrm{L}} \rho_{\boldsymbol{k}}^{\mathrm{s}} \mid \mathcal{T}_{+} \boldsymbol{v}_{s}\right\rangle-\frac{1}{3}\left\langle j_{-\boldsymbol{k}}^{\mathrm{L}} \rho_{\boldsymbol{k}}^{\mathrm{s}} \mid \boldsymbol{v}_{s}\right\rangle\left\langle\boldsymbol{v}_{s} \mid \mathcal{T}_{+} \boldsymbol{v}_{s}\right\rangle \\
& =\hat{\boldsymbol{k}}\left\langle j_{\boldsymbol{k}}^{\mathrm{L}} \mid \mathcal{T}_{+} j_{\boldsymbol{k}}^{\mathrm{sL}}\right\rangle-\mathrm{i} \frac{1+\varepsilon}{3} \omega_{\mathrm{E}} \hat{\boldsymbol{k}}\left\langle j_{\boldsymbol{k}}^{\mathrm{L}} \mid j_{\boldsymbol{k}}^{\mathrm{sL}}\right\rangle .
\end{aligned}
$$

This shows that the left and the right vertex are identical. With $\left\langle j_{\boldsymbol{k}}^{\mathrm{L}} \mid j_{\boldsymbol{k}}^{\mathrm{sL}}\right\rangle=1 / N$ and $\left\langle j_{\boldsymbol{k}}^{\mathrm{L}} \mid \mathcal{T}_{+} j_{\boldsymbol{k}}^{\mathrm{sL}}\right\rangle=\nu_{k} / N$, where $\nu_{k}$ was determined in [23], equation (16a) follows.

\section{Appendix A.2. Transverse}

Starting like in the longitudinal case, we find

$$
\left\langle\boldsymbol{j}_{-\boldsymbol{k}}^{\mathrm{T}} \rho_{\boldsymbol{k}}^{\mathrm{s}} \mathcal{Q} \mid \mathcal{L}_{+} \boldsymbol{v}_{s}\right\rangle=\left\langle\boldsymbol{j}_{\boldsymbol{k}}^{\mathrm{T}} \mid \mathcal{T}_{+} \boldsymbol{j}_{\boldsymbol{k}}^{\mathrm{sT}}\right\rangle-\mathrm{i} \frac{1+\varepsilon}{3} \omega_{\mathrm{E}}\left\langle\boldsymbol{j}_{\boldsymbol{k}}^{\mathrm{T}} \mid \boldsymbol{j}_{\boldsymbol{k}}^{\mathrm{sT}}\right\rangle
$$

The proof that the left and right vertices are identical is completely analogous to the discussion above.

We have $\left\langle\boldsymbol{j}_{\boldsymbol{k}}^{\mathrm{T}} \mid \boldsymbol{j}_{\boldsymbol{k}}^{\mathrm{sT}}\right\rangle=2 / N$ and $\left\langle\boldsymbol{j}_{\boldsymbol{k}}^{\mathrm{T}} \mid \mathcal{T}_{+} \boldsymbol{j}_{\boldsymbol{k}}^{\mathrm{sT}}\right\rangle=\left\langle\boldsymbol{j}_{\boldsymbol{k}} \mid \mathcal{T}_{+} \boldsymbol{j}_{\boldsymbol{k}}^{\mathrm{s}}\right\rangle-\left\langle j_{\boldsymbol{k}}^{\mathrm{L}} \mid \mathcal{T}_{+} j_{\boldsymbol{k}}^{\mathrm{sL}}\right\rangle . \quad$ With $\boldsymbol{v}=\left(\boldsymbol{v}_{1}-\boldsymbol{v}_{s}\right) / \sqrt{2}$ and $\boldsymbol{r}=\boldsymbol{r}_{1}-\boldsymbol{r}_{s}$ we write

$$
\left\langle\boldsymbol{j}_{\boldsymbol{k}} \mid \mathcal{T}_{+} \boldsymbol{j}_{\boldsymbol{k}}^{\mathrm{s}}\right\rangle=\mathrm{i} \frac{1+\varepsilon}{2} \sqrt{2}\left\langle(\hat{\boldsymbol{r}} \cdot \boldsymbol{v})^{3} \Theta(-\hat{\boldsymbol{r}} \cdot \boldsymbol{v}) \delta(r-d)\left(e^{-\mathrm{i} \boldsymbol{q} \cdot \boldsymbol{r}}-1\right)\right\rangle
$$

where $\sqrt{2}\left\langle(\hat{\boldsymbol{r}} \cdot \boldsymbol{v})^{3} \Theta(-\hat{\boldsymbol{r}} \cdot \boldsymbol{v})\right\rangle=-2 / \sqrt{\pi}$ and

$$
\begin{aligned}
\left\langle\delta(r-d)\left(e^{-\mathrm{i} \boldsymbol{q} \cdot \boldsymbol{r}}-1\right)\right\rangle & =\frac{2 \pi d^{2} \chi}{V} \int_{0}^{\pi} \mathrm{d} \vartheta \sin \vartheta\left(e^{-\mathrm{i} q d \cos \vartheta}-1\right) \\
& =-24 \frac{\varphi \chi}{d N}\left[1-j_{0}(q d)\right],
\end{aligned}
$$

i.e.,

$$
\left\langle\boldsymbol{j}_{\boldsymbol{k}} \mid \mathcal{T}_{+} \boldsymbol{j}_{\boldsymbol{k}}^{\mathrm{s}}\right\rangle=2 \mathrm{i} \frac{1+\varepsilon}{2 N} \omega_{\mathrm{E}}\left[1-j_{0}(q d)\right]
$$

Using suitable relations between spherical Bessel functions, equation (16b) follows. 


\section{Appendix B. Some Integrals}

All the integrals needed for $m_{\rho, \mathrm{L}}(t \rightarrow \infty)$ can be expressed as derivatives of

$$
I(c, G ; t):=\int_{0}^{\infty} \mathrm{d} k \cos (c k t) e^{-G k^{2} t}=\frac{1}{2} \sqrt{\frac{\pi}{G t}} \exp \left(-c^{2} t / 4 G\right),
$$

where the second equality is given in [29]. Then we have

$$
\begin{gathered}
\int_{0}^{\infty} \mathrm{d} k k^{2} \cos (c k t) e^{-G k^{2} t}=-\frac{1}{t} \frac{\partial I}{\partial G}=-\frac{1}{4} I(c, G ; t) \frac{c^{2} t-2 G}{G^{2} t}, \\
\int_{0}^{\infty} \mathrm{d} k k^{4} \cos (c k t) e^{-G k^{2} t}=\frac{1}{t^{2}} \frac{\partial^{2} I}{\partial G^{2}} \\
=\frac{1}{16} I(c, G ; t) \frac{12 G^{2}-12 c^{2} G t+c^{4} t^{2}}{G^{4} t^{2}},
\end{gathered}
$$

and,

$$
\int_{0}^{\infty} \mathrm{d} k k^{3} \sin (c k t) e^{-G k^{2} t}=\frac{1}{t^{2}} \frac{\partial^{2} I}{\partial G \partial c}=\frac{1}{8} I(c, G ; t) c \frac{c^{2} t-6 G}{G^{3} t} .
$$

In particular,

$$
2 c G \frac{1}{t^{2}} \frac{\partial^{2} I}{\partial G \partial c}+c^{2} \frac{1}{t} \frac{\partial I}{\partial G}=\frac{\sqrt{\pi}}{2} \frac{c^{2}}{G}(G t)^{-3 / 2} \exp \left(-c^{2} t / 4 G\right) .
$$

\section{References}

[1] B. J. Alder and T. E. Wainwright. Velocity autocorrelations for hard spheres. Phys. Rev. Lett., 18(23):988-990, 1967.

[2] B. J. Alder and T. E. Wainwright. Decay of the velocity autocorrelation function. Phys. Rev. A, 1(1):18-21, 1970.

[3] Y. Pomeau and P. Résibois. Time dependent correlation functions and mode-mode coupling theories. Phys. Rep., 19(2):63-139, 1975.

[4] P. Kovtun and L. G. Yaffe. Hydrodynamic fluctuations, long-time tails, and supersymmetry. Phys. Rev. D, 68(2):025007, 2003.

[5] R. Golestanian. Anomalous diffusion of symmetric and asymmetric active colloids. Phys. Rev. Lett., 102(18):188305, 2009.

[6] S. R. Ahmad and S. Puri. Velocity distributions and aging in a cooling granular gas. Phys. Rev. E, 75(3):031302, 2007.

[7] H. Hayakawa and M. Otsuki. Long-time tails in freely cooling granular gases. Phys. Rev. E, 76(5):051304, 2007.

[8] M. Otsuki and H. Hayakawa. Unified description of long-time tails and long-range correlation functions for sheared granular liquids. Eur. Phys. J. Spec. Top., 179(1):179-195, 2009.

[9] A. V. Orpe, V. Kumaran, K. A. Reddy, and A. Kudrolli. Fast decay of the velocity autocorrelation function in dense shear flow of inelastic hard spheres. Europhys. Lett., 84(6):64003, 2008.

[10] V. Kumaran. Dynamics of a dilute sheared inelastic fluid. i. hydrodynamic modes and velocity correlation functions. Phys. Rev. E, 79(1):011301, 2009.

[11] A. Fiege, T. Aspelmeier, and A. Zippelius. Long-time tails and cage effect in driven granular fluids. Phys. Rev. Lett., 102(9):098001, 2009.

[12] P. Résibois. Approximate kinetic theory of hard-sphere fluids near equilibrium: II. A quasihydrodynamic approximation for the velocity autocorrelation function. J. Stat. Phys., 13(5):393-426, 1975. 
[13] P. M. Furtado, G. F. Mazenko, and S. Yip. Effects of correlated collisions on atomic diffusion in a hard-sphere fluid. Phys. Rev. A, 14(2):869, 1976.

[14] J. Bosse, W. Götze, and A. Zippelius. Velocity-autocorrelation spectrum of simple classical liquids. Phys. Rev. A, 18(3):1214, 1978.

[15] R. I. Cukier and J. R. Mehaffey. Kinetic theory of self-diffusion in a hard-sphere fluid. Phys. Rev. A, 18(3):1202, 1978.

[16] L. Sjögren and A. Sjölander. Kinetic theory of self-motion in monatomic liquids. J. Phys. C, 12:4369-4392, 1979.

[17] T. R. Kirkpatrick and J. C. Nieuwoudt. Mode-coupling theory of the intermediate-time behavior of the velocity autocorrelation function. Phys. Rev. A, 33:2658-2662, 1986.

[18] M. H. Ernst and A. Weyland. Long time behaviour of the velocity auto-correlation function in a Lorentz gas. Phys. Lett. A, 34(1):39-40, 1971.

[19] F. Höfling and T. Franosch. Crossover in the slow decay of dynamic correlations in the lorentz model. Phys. Rev. Lett., 98(14):140601, 2007.

[20] M. Otsuki and H. Hayakawa. Long-time tails for sheared fluids. J. Stat. Mech., 2009(08):L08003, 2009.

[21] P. K. Haff. Grain flow as a fluid-mechanical phenomenon. J. Fluid Mech., 134:401-430, 1983.

[22] P. Espanol and P. Warren. Statistical mechanics of dissipative particle dynamics. Europhys. Lett., 30:191-196, 1995.

[23] W. T. Kranz, M. Sperl, and A. Zippelius. Glass transition in driven granular fluids: A modecoupling approach. Phys. Rev. E, 87(2):022207, 2013.

[24] A. R. Altenberger. On the calculation of the classical Liouville operator for the step-type interparticle interaction. Physica A, 80(1):46 - 62, 1975.

[25] J. P. Boon and S. Yip. Molecular Hydrodynamics. Dover Publications, New York, 1992.

[26] H. Mori. A continued-fraction representation of the time-correlation functions. Prog. Theor. Phys., 34:399-416, 1965.

[27] J.-P. Hansen and I. R. McDonald. Theory of Simple Liquids. Academic Press, Amsterdam, 3 edition, 2006.

[28] M. Sperl, W. T. Kranz, and A. Zippelius. Single-particle dynamics in dense granular fluids under driving. Europhys. Lett., 98(2):28001, 2012.

[29] A. Jeffrey and D. Zwillinger, editors. Gradshteyn and Ryzhik's Table of Integrals, Series, and Products. Academic Press, San Diego, 6th edition, 2000.

[30] I. Goldhirsch. Rapid granular flows. Annu. Rev. Fluid Mech., 35(1):267-293, 2003.

[31] K. Vollmayr-Lee, T. Aspelmeier, and A. Zippelius. Hydrodynamic correlation functions of a driven granular fluid in steady state. Phys. Rev. E, 83(1):011301, 2011.

[32] T. P. C. van Noije, M. H. Ernst, E. Trizac, and I. Pagonabarraga. Randomly driven granular fluids: Large-scale structure. Phys. Rev. E, 59(4):4326-4341, 1999.

[33] V. Garzó and J. M. Montanero. Transport coefficients of a heated granular gas. Physica A, 313(3-4):336-356, 2002.

[34] T. Franosch and W. Götze. Mode-coupling theory for the shear viscosity in supercooled liquids. Phys. Rev. E, 57(5):5833, 1998. 